hep-ph/0601203

$25^{\text {th }}$ January 2006

\title{
Analysis of charmonium production at fixed-target experiments in the NRQCD approach
}

\author{
F. Maltoni, \\ Institut de Physique Théorique, Université Catholique de Louvain \\ Chemin du Cyclotron, 2, B-1348 Louvain-la-Neuve, Belgium \\ J. Spengler, \\ Max-Planck-Institut für Kernphysik, D-69117 Heidelberg, Germany ${ }^{1}$
}

M. Bargiotti, A. Bertin, M. Bruschi, S. De Castro, L. Fabbri, P. Faccioli, B. Giacobbe, F. Grimaldi, I. Massa, M. Piccinini, N. Semprini-Cesari, R. Spighi, M. Villa, A. Vitale and A. Zoccoli

Dipartimento di Fisica dell'Università di Bologna

and INFN Sezione di Bologna, I-40126 Bologna, Italy

\begin{abstract}
We present an analysis of the existing data on charmonium hadro-production based on nonrelativistic QCD (NRQCD) calculations at the next-to-leading order (NLO). All the data on $J / \psi$ and $\psi(2 S)$ production in fixed-target experiments and on $p p$ collisions at low energy are included. We find that the amount of color octet contribution needed to describe the data is about $1 / 10$ of that found at the Tevatron.
\end{abstract}

\section{Introduction}

The production of charmonium and bottomonium states in high-energy collisions has always been the subject of considerable interest [1. From the experimental point of view, charmonium decays into lepton pairs offer very clean signatures that are used not only for triggering and calibration but also to perform important physics studies. The decay $B \rightarrow J / \psi+X$, for instance, provides an easy handle to QCD studies of $b$ production and to the precise determination of some of the CKM parameters, such as $\sin 2 \beta$.

The importance of quarkonium is widely recognized also by the theoretical community. Charmonium and bottomonium states offer a unique laboratory for testing our understanding of QCD, and in particular of the interplay between the perturbative and non-perturbative regimes, which describe the physics of heavy-quark creation and that of bound state formation, respectively. The so-called color-singlet model [2, 3] has been superseded by a rigorous framework, based on the use of non-relativistic QCD (NRQCD) 4, an effective field theory that consistently includes relativistic corrections and provides a solid ground for accurate theoretical analyses.

\footnotetext{
${ }^{1}$ Supported by the Bundesministerium für Bildung und Forschung, FRG, under contract numbers 05-7MP25I.
} 
However, despite the theoretical developments and successes, not all the predictions of the NRQCD factorization approach have been firmly established. The first example is the universality of the nonpertubative matrix elements, on which the predictive power of this approach relies. Measurements at the Tevatron in proton-proton collisions suggest larger values for the color-octet matrix elements than those obtained at HERA in electron-proton collisions [5. Even more problematic is the measurement of $J / \psi$ polarization at the Tevatron. NRQCD predicts a sizable transverse polarization for $J / \psi$ 's at high- $p_{T}$, in contrast with the latest data that now clearly indicate that $J / \psi$ 's are not transversely polarized [6]. While there is no quantitative and accepted explanation for this behaviour, it is generally argued that because the quarkonium mass is still not very large with respect to the QCD scale, in particular for the charmonium system, non-factorizable corrections may not be suppressed enough and/or the expansions in NRQCD may not converge very well.

In the light of the present uncertain status, detailed studies on the range of applicability of the NRQCD approach, above all for charmonium states, are certainly welcome. In this work, we perform the NRQCD analysis of charmonium production data as obtained from experiments at fixed-target (with the exclusion of that induced by pion beams) and from $p p$ collisions at low energy. Our purpose is twofold. First, we present an up-to-date collection of the experimental data on charmonium production in fixed-target experiments. Second, we study whether data are consistent with the NRQCD approach and in particular we extract information on the color-octet contributions, to be compared with that obtained from other experiments. Our analysis represents an improvement with respect to the previous studies presented in Refs. 7, 8, both in terms of accuracy of the theoretical predictions and for the more complete and comprehensive treatment of the experimental data. The outline of the paper is as follows. In the next section, we briefly review the framework of the NRQCD approach and state the theoretical results and assumptions that enter into our predictions. In Section 3 we discuss the experimental data. In Section 4 we describe the fit strategy and present the results. We draw our conclusions in the last section.

\section{The NRQCD approach}

In the NRQCD approach, the cross section for the production of a quarkonium state $H$ in a nucleonnucleon interaction is expressed as a sum of terms, each of which factors into a short-distance coefficient and a long-distance matrix element:

$$
\sigma(p p \rightarrow H+X)=\sum_{i, j} \int d x_{1} d x_{2} f_{i / p} f_{j / p} \sum_{n} \hat{\sigma}(i j \rightarrow Q \bar{Q}[n]+x)\left\langle\mathcal{O}^{H}[n]\right\rangle,
$$

where the indexes $i, j$ run over all the partonic species and $n$ denotes the color, spin and angular momentum state of an intermediate $Q \bar{Q}$ pair. The short-distance cross section $\hat{\sigma}$ can be calculated as a perturbative expansion in the strong coupling $\alpha_{s}$. The NRQCD matrix elements $\left\langle\mathcal{O}^{H}[n]\right\rangle$ (see Ref. [4] for their definition) are related to the non-perturbative transition probabilities from the $Q \bar{Q}$ state $n$ into the quarkonium $H$. They scale according to a definite power of the intrinsic heavy-quark velocity $v$ $\left(v^{2} \sim 0.3\right.$ for charmonium and $v^{2} \sim 0.1$ for bottomonium) 9]. The general expression (11) is thus a double expansion in powers of $\alpha_{s}$ and $v$. While a formal and general proof of Eq. (11) is still lacking, it has been recently shown [10] that it holds for high- $p_{T}$ quarkonium production up to its two-loop description. In this work, we simply assume that soft effects do not spoil factorization and Eq. (11) holds true also for total cross sections.

The color-singlet short distance coefficients for spin singlet $S$-wave $\left({ }^{1} S_{0}^{[1]}\right), P$-waves and all the leading

color-octet coefficients are known at NLO for both photon-proton and proton-proton collisions [11, 


\begin{tabular}{cccc}
\hline \hline$H$ & $\left\langle\mathcal{O}_{1}^{H}\right\rangle$ & $\left\langle\mathcal{O}_{8}^{H}\left[{ }^{3} S_{1}\right]\right\rangle$ & $\left\langle\mathcal{O}_{8}^{H}\left[{ }^{1} S_{0}^{(8)}\right]\right\rangle=\left\langle\mathcal{O}_{8}\left[{ }^{3} P_{0}^{(8)}\right]\right\rangle / m_{c}^{2}$ \\
\hline$J / \psi$ & $1.16 \mathrm{GeV}^{3}$ & $1.19 \cdot 10^{-2} \mathrm{GeV}^{3}$ & $1.0 \cdot 10^{-2} \mathrm{GeV}^{3}$ \\
$\psi(2 S)$ & $0.76 \mathrm{GeV}^{3}$ & $0.50 \cdot 10^{-2} \mathrm{GeV}^{3}$ & $0.42 \cdot 10^{-2} \mathrm{GeV}^{3}$ \\
$\chi_{c 0}$ & $0.11 \mathrm{GeV}^{5}$ & $0.31 \cdot 10^{-2} \mathrm{GeV}^{3}$ & -- \\
\hline \hline
\end{tabular}

Table 1: Reference NRQCD matrix elements for charmonium production. The color-singlet matrix elements are taken from the potential model calculation of [14, 15]. The color-octet matrix elements have been extracted from the CDF data [16] in Ref. [17.

12. The color-singlet coefficient for ${ }^{3} S_{1}^{[1]}$ is known at NLO only for photon-proton collisions [13]. In this respect our analysis cannot be considered as fully at NLO and should be updated once the NLO calculation for the color singlet term will be available. On the other hand, this is not an important limitation to our results, as will be made clear in the following.

The non-perturbative matrix elements have to be extracted from the data. For the color-singlet terms this is straightforward. It can be easily shown that, up to relativistic corrections of order $v^{4}$, they can be related to those appearing in the corresponding decay rates and therefore can be extracted from measurements of decay widths. On the other hand, color-octet matrix elements can only be extracted from production processes, such as photoproduction or hadroproduction. The factorization hypothesis implies that the values extracted from different experiments should be universal. Table 1 shows the results of a fit performed on the CDF charmonium data [16], providing the leading color octet terms for $J / \psi, \psi(2 S)$ and $\chi_{c J}$ production at the Tevatron energy [17. In the case of $S$-waves, the fact that transverse momentum distributions coming from CP-even states $\left({ }^{1} S_{0}^{[8]}\right.$ and $\left.{ }^{3} P_{J}^{[8]}\right)$ and the ${ }^{3} S_{1}^{[8]}$ have different shapes, has been exploited to obtain information on their relative size. An equally detailed information cannot be extracted from fixed-target results, normally limited to the total production rates.

The analysis performed here is based on a code implementing the NLO calculations of Ref. [12. For the theoretical inputs, we make the following choices. We use $\mu_{0}=2 m_{c}$ with $m_{c}=1.5 \mathrm{GeV}$ as our central value for the renormalization, factorization and NRQCD scales (all taken equal). We estimate the associated uncertainty by varying the scales between $\mu_{0}$ and $4 \mu_{0}$. The strong coupling constant $\alpha_{S}\left(m_{Z}\right)$ is tuned to the one used in the PDF sets, i.e., CTEQ6m [18] and MRST2002nlo [19. We exploit spin symmetry to reduce the number of independent non-perturbative matrix elements,

$$
\begin{aligned}
\left\langle\mathcal{O}_{8}^{\psi}\left({ }^{3} P_{J}\right)\right\rangle & =(2 J+1)\left\langle\mathcal{O}_{8}^{\psi}\left({ }^{3} P_{0}\right)\right\rangle \\
\left\langle\mathcal{O}_{8}^{\chi_{c J}}\left({ }^{3} S_{1}\right)\right\rangle & =(2 J+1)\left\langle\mathcal{O}_{8}^{\chi_{c 0}}\left({ }^{3} S_{1}\right)\right\rangle \\
\left\langle\mathcal{O}_{1}^{\chi_{c J}}\left({ }^{3} P_{J}\right)\right\rangle & =(2 J+1)\left\langle\mathcal{O}_{1}^{\chi_{c 0}}\left({ }^{3} P_{0}\right)\right\rangle
\end{aligned}
$$

and consider only leading color octet corrections. We take the non-perturbative matrix elements collected in Tab. 1 as our reference values. ${ }^{2}$ Color-singlet matrix elements are kept fixed. For the color-octet matrix elements we adopt the relative normalization as that obtained from Tevatron measurements,

\footnotetext{
${ }^{2}$ See also Ref. [20] for a more recent analysis.
} 
but for the $S$-wave color-octet matrix-element two overall multiplicative numbers $\lambda_{J / \psi}$ and $\lambda_{\psi(2 S)}$ are introduced to be fitted with the fixed-target data. The term $\left\langle\mathcal{O}_{8}^{\chi_{c 0}}\left({ }^{3} S_{1}\right)\right\rangle$ is left fixed to its reference value extracted from the Tevatron data. With the above assumptions, the data are fitted with only two free parameters, the $\lambda$ 's, which can be interpreted as the fractions of the "overall Tevatron octet contribution" for $J / \psi$ and $\psi(2 S)$ necessary to explain the fixed-target data.

The following theoretical expressions for the cross sections are used:

$$
\begin{aligned}
& \sigma_{\psi(2 S)}=\sigma_{\psi(2 S)}^{\mathrm{D}} \\
& \sigma_{J / \psi}=\sigma_{J / \psi}^{\mathrm{D}}+\sum_{J=0}^{2} \operatorname{Br}\left(\chi_{c J} \rightarrow J / \psi \gamma\right) \sigma_{\chi_{c J}}^{\mathrm{D}}+\operatorname{Br}(\psi(2 S) \rightarrow J / \psi X) \sigma_{\psi(2 S)}, \\
& R_{\psi}=\frac{\sigma_{\psi(2 S)}}{\sigma_{J / \psi}}
\end{aligned}
$$

where the superscript D refers to the direct contribution. For the branching ratios we use [21]

$$
\begin{aligned}
\operatorname{Br}(\psi(2 S) \rightarrow J / \psi X) & =57.6 \% \\
\operatorname{Br}\left(\chi_{c 0} \rightarrow J / \psi \gamma\right) & =1.18 \% \\
\operatorname{Br}\left(\chi_{c 1} \rightarrow J / \psi \gamma\right) & =31.6 \% \\
\operatorname{Br}\left(\chi_{c 2} \rightarrow J / \psi \gamma\right) & =20.2 \%
\end{aligned}
$$

\section{Present experimental situation}

The measurements of $J / \psi$ hadroproduction have been performed in a time period spanning about thirty years. Over such a long period, several different experimental techniques have been used and different input information was available at the time of the measurements. Therefore, comparing results of different experiments on an equal footing requires an update of the published numbers on several aspects. For example, the charmonium branching ratios have changed with time and the treatment of the nuclear effects are not homogeneous. In our compilations, we update all the measurements with the current best knowledge of branching ratios and nuclear effects.

\subsection{Compilation of $J / \psi$ cross sections}

The cross section for $J / \psi$ production on a nuclear target of mass number A is parametrized as

$$
\sigma_{J / \psi}^{p A}=\sigma_{J / \psi} \cdot A^{\alpha}
$$

For this comparison the most precise measurement of $\alpha=0.96 \pm 0.01\left[22\right.$ at $x_{F}(J / \psi) \simeq 0$ is used, assuming its independence of the cms-energy. If an experiment has published cross sections obtained from different targets, Eq. (5) is applied to obtain a combined result. This is the case for the experiments CERN-PS [23] (H, C, W), NA50 [37] (Be, Al, Cu, Ag and W) and HERA-B [43] (C, Ti and W). As suggested in Ref. [36], we combine the results of NA51 [35] (H, D targets) and NA38 [36] $(\mathrm{C}, \mathrm{Al}, \mathrm{Cu}$, $\mathrm{W})$.

The results collected in Tab. 2 are updated with the latest branching fractions [21] (5.88 $\pm 0.10 \%$ for $J / \psi \rightarrow \mu^{+} \mu^{-}$and $5.93 \pm 0.10 \%$ for $\left.J / \psi \rightarrow \mathrm{e}^{+} \mathrm{e}^{-}\right)$. If only the forward cross section $\left(x_{F}(J / \psi)>0\right)$ is given, we assume a symmetric $d \sigma_{J / \psi} / d x_{F}$ distribution and multiply the forward cross section by two to obtain the total cross section. The cross sections of NA38, NA50 and NA51 are only quoted for a limited 
phase-space. In order to obtain total cross sections, the extrapolation discussed in the NA51 paper is performed [35.

Unless it is clearly stated in the publications, we assume that the quoted uncertainties on branching ratio and atomic mass dependence were taken into account in the systematic uncertainty. Therefore we consistently update them in Tab. 2

The updated results for mid-rapidity and total cross sections are displayed in Fig. 1].
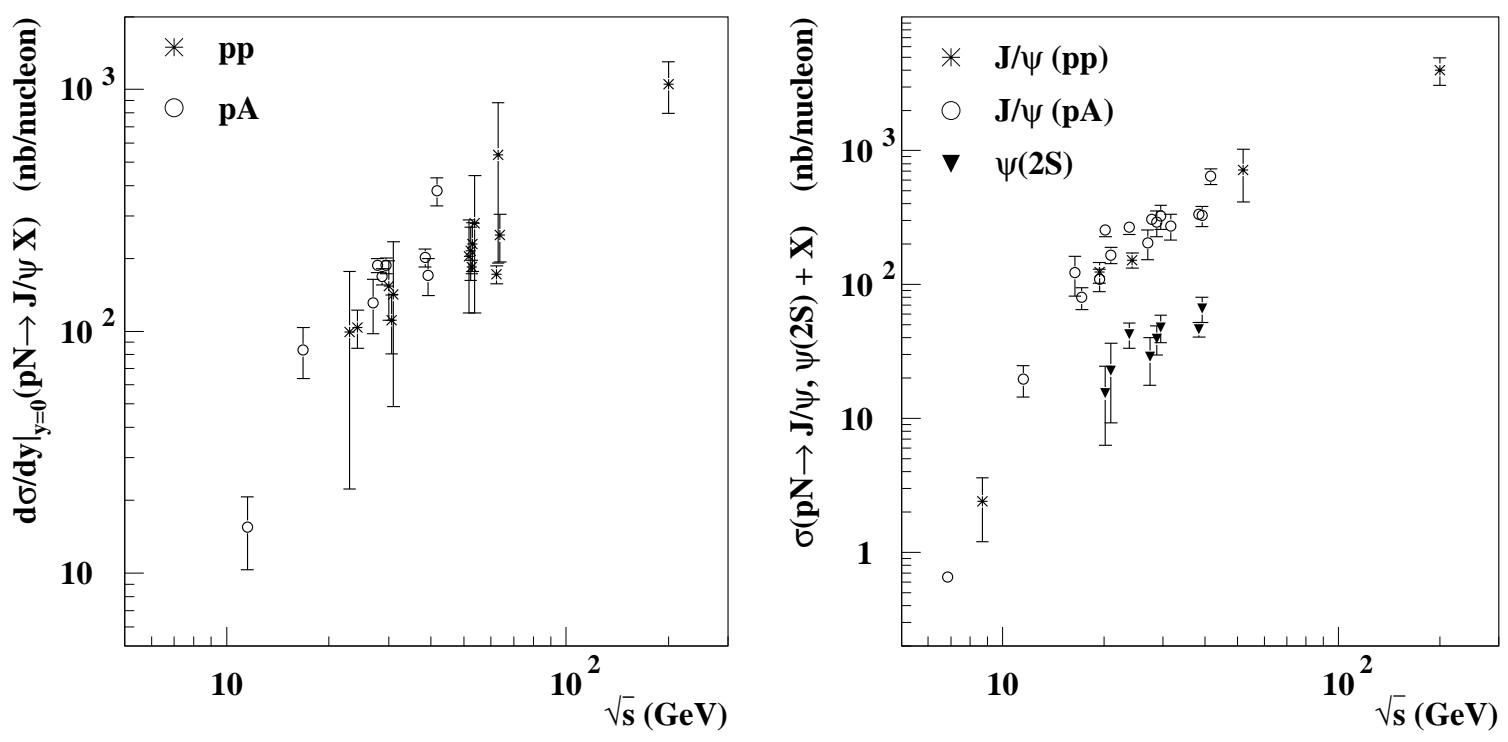

Figure 1: Production cross sections in proton-induced interactions from Tab. 2 as a function of the cmsenergy. Proton-proton (pp) and proton-nucleus (pA) measurements are indicated by different symbols. Left: differential cross sections $d \sigma_{p N} / d y$ at $y=0$; right: total cross sections for $J / \psi$ and $\psi(2 S)$ production.

\subsection{Compilation of $\psi(2 S)$ cross sections}

The procedure described in the previous section is applied to the published $\psi(2 S)$ cross sections using the most recent branching fractions [21] $\left(0.73 \pm 0.08 \%\right.$ for $\psi(2 S) \rightarrow \mu^{+} \mu^{-}$and $0.755 \pm 0.031 \%$ for $\left.\psi(2 S) \rightarrow \mathrm{e}^{+} \mathrm{e}^{-}\right)$and $\alpha=0.934 \pm 0.010[22]$ at $x_{F}(\psi(2 S)) \simeq 0$.

The results on the $\psi(2 S)$ cross sections (see Fig. 11) and the ratios between $\psi(2 S)$ and $J / \psi$ cross sections are listed in Tab. 3 .

\subsection{Comments on the available data}

The $J / \psi$ cross sections have been usually measured on large samples by triggering on dilepton decays. Therefore the most precise data have uncertainties dominated by systematic errors. The measurements show a good overall consistency, even though some of the results are hardly compatible. For instance, the two results at $20.6 \mathrm{GeV}$ (E331 [28] and E444 [29]) differ by roughly $2 \sigma$. The E705 [31] result at 23.8 $\mathrm{GeV}$ exceeds the UA6 32] one at $24.3 \mathrm{GeV}$ by more than $3 \sigma$, in contrast with the expectation (and the general trend) that the cross section should increase with the cms-energy. This is an indication that 


\begin{tabular}{|l|c|c|c|c|}
\hline Experiment & Reaction & $\begin{array}{c}\sqrt{\mathrm{s}} \\
(\mathrm{GeV})\end{array}$ & $\begin{array}{c}\left.\frac{d \sigma_{J / \psi}}{d y}\right|_{y=0} \\
(\mathrm{nb} / \text { nucleon })\end{array}$ & $\begin{array}{c}\sigma_{J / \psi} \\
(\mathrm{nb} / \text { nucleon })\end{array}$ \\
\hline \hline CERN-PS [23] & $\mathrm{pA}$ & 6.8 & & $0.65 \pm 0.06$ \\
\hline WA39 [24] & $\mathrm{pp}$ & 8.7 & & $2.4 \pm 1.2$ \\
\hline IHEP [25] & $\mathrm{pBe}$ & 11.5 & $16 \pm 5.2$ & $20 \pm 5.2$ \\
\hline E331 [26] & $\mathrm{pBe}$ & 16.8 & $84 \pm 20$ & $122 \pm 40$ \\
\hline NA3 [27] & $\mathrm{pPt}$ & 16.8 & & $80 \pm 15$ \\
\hline NA3 [27] & $\mathrm{pPt}$ & 19.4 & & $110 \pm 21$ \\
\hline NA3 [27] & $\mathrm{pp}$ & 19.4 & & $124 \pm 22$ \\
\hline E331 [28] & $\mathrm{pC}$ & 20.6 & & $256 \pm 30$ \\
\hline E444 [29] & $\mathrm{pC}$ & 20.6 & & $166 \pm 23$ \\
\hline ISR [30] & $\mathrm{pp}$ & 23.0 & $100 \pm 77$ & \\
\hline E705 [31] & $\mathrm{pLi}$ & 23.8 & & $267 \pm 30$ \\
\hline UA6 [32] & $\mathrm{pp}$ & 24.3 & $104 \pm 19$ & $152 \pm 20$ \\
\hline E288[33] & $\mathrm{pBe}$ & 27.4 & $131 \pm 33$ & $204 \pm 51$ \\
\hline E595[34] & $\mathrm{pFe}$ & 27.4 & $187 \pm 12$ & $306 \pm 18$ \\
\hline NA38/51 35], 36] & $\mathrm{pA}$ & 29.1 & $169 \pm 13$ & $292 \pm 64$ \\
\hline NA50 [37] & $\mathrm{pA}$ & 29.1 & $188 \pm 14$ & $325 \pm 67$ \\
\hline ISR [38] & $\mathrm{pp}$ & 30 & $154 \pm 42$ & \\
\hline ISR [39] & $\mathrm{pp}$ & 30.6 & $111 \pm 30$ & \\
\hline ISR [30] & $\mathrm{pp}$ & 31 & $142 \pm 93$ & \\
\hline E672/706 40] & $\mathrm{pBe}$ & 31.6 & & $274 \pm 60$ \\
\hline E771 [41] & $\mathrm{pSi}$ & 38.8 & $202 \pm 17$ & $333 \pm 25$ \\
\hline E789 [42] & $\mathrm{pAu}$ & 38.8 & $170 \pm 30$ & $327 \pm 56$ \\
\hline HERA-B [43] & $\mathrm{pA}$ & 41.6 & $392 \pm 51$ & $663 \pm 87$ \\
\hline ISR [44] & $\mathrm{pp}$ & 52 & $204 \pm 85$ & $716 \pm 303$ \\
\hline ISR [45] & $\mathrm{pp}$ & 52 & $216 \pm 54$ & \\
\hline ISR [39] & $\mathrm{pp}$ & 52.4 & $185 \pm 12$ & \\
\hline ISR [38] & $\mathrm{pp}$ & 53 & $229 \pm 52$ & \\
\hline ISR [30] & $\mathrm{pp}$ & 53 & $280 \pm 161$ & \\
\hline ISR [39] & $\mathrm{pp}$ & 62.7 & $172 \pm 15$ & \\
\hline ISR [30] & $\mathrm{pp}$ & 63 & $250 \pm 56$ & \\
\hline ISR [38] & 200 & $1051 \pm 255$ & $4000 \pm 938$ \\
\hline PHENIX [46] & & & \\
\hline
\end{tabular}

Table 2: Updated differential $\left(d \sigma_{J / \psi} / d y\right.$ at $\left.y=0\right)$ and total $\left(\sigma_{J / \psi}\right)$ production cross sections in protoninduced interactions. The pA symbol in the second column indicates that the cross section value is obtained by fitting different target materials.

probably in some measurements the systematic uncertainties coming from triggering effects or luminosity determination have been underestimated.

The $\psi(2 S)$ cross sections are usually estimated on the same data sample of the $\sigma_{J / \psi}$, but are poorer in statistics (by a factor around 60). Therefore they are less sensitive to the accurate determination of 


\begin{tabular}{|l|c|c|c|c|}
\hline Experiment & Reaction & $\begin{array}{c}\sqrt{\mathrm{s}} \\
(\mathrm{GeV})\end{array}$ & $\begin{array}{c}\sigma_{\psi(2 S)} \\
(\mathrm{nb} / \text { nucleon })\end{array}$ & $\begin{array}{c}\sigma_{\psi(2 S)} / \sigma_{J / \psi} \\
\left(R_{\psi}\right)\end{array}$ \\
\hline \hline E331[28] & $\mathrm{pC}$ & 20.6 & $15.4 \pm 9.1$ & $0.060 \pm 0.035$ \\
\hline E444[29] & $\mathrm{pC}$ & 20.6 & $22.8 \pm 13.5$ & $0.137 \pm 0.079$ \\
\hline E705[31] & $\mathrm{pLi}$ & 23.8 & $42.5 \pm 9.0$ & $0.159 \pm 0.029$ \\
\hline E288[33] & $\mathrm{pBe}$ & 27.4 & $28.9 \pm 11.3$ & $0.141 \pm 0.042$ \\
\hline NA38/51[35], [36] & $\mathrm{pA}$ & 29.1 & $39.3 \pm 9.6$ & $0.135 \pm 0.015$ \\
\hline NA50[37] & $\mathrm{pA}$ & 29.1 & $47.1 \pm 10.9$ & $0.145 \pm 0.017$ \\
\hline E771 [4]] & $\mathrm{pSi}$ & 38.8 & $46.3 \pm 5.7$ & $0.139 \pm 0.020$ \\
\hline E789 [4]] & $\mathrm{pAu}$ & 38.8 & $66.1 \pm 14.1$ & $0.202 \pm 0.055$ \\
\hline
\end{tabular}

Table 3: Updated total production cross sections of $\psi(2 S)$ and ratios of $\psi(2 S)$ to $J / \psi$ cross sections in proton-induced interactions.

the systematic effects. Within the quoted uncertainties, there is a good internal consistency among the different measurements.

In the $R_{\psi}$ cross section ratios, the systematic effects on luminosity or trigger mainly cancel out and the final uncertainty is usually dominated by the $\psi(2 S)$ statistics. The measurements are all compatible.

Since the measurements on the polarization are all compatible at $3 \sigma$ with the assumption of no polarization (40, 41, 47]), possible effects of the polarization in the $J / \psi$ or $\psi(2 S)$ decays are always neglected and in the evaluation of the cross sections the polarization is assumed to be negligible for both charmonium states. It should be noted that if any polarization is present (expecially for $\psi(2 S)$ where a precise information is lacking) some results might be affected by a systematic uncertainty larger than that quoted.

\section{Fit results}

The cross sections values obtained from NRQCD calculations at NLO, Eqs. (11) and (3), have been used to fit the experimental results summarized in Tabs. 2, 3,

The ratio of the two color octet matrix elements for $J / \psi$ and $\psi(2 S)$ production to the Tevatron ones $\left(\lambda_{J / \psi}\right.$ and $\lambda_{\psi(2 S)}$ respectively) are used to fit the theoretical predictions to the experimental values.

Since the cross section depends on the product of the matrix elements and the PDF's as shown in Eq. (11), a change in the PDF can influence the result of the fit on the two color octet matrix elements. To estimate such effect we used two different PDF sets, the CTEQ6m [18] and the MRST2002nlo [19].

The data considered in the fit (Tabs. 2] and 3) include 21 results on the total $J / \psi$ cross section (cms-energy range: [6.7 : 200] GeV), three $\psi(2 S)$ cross sections (NA50 [37, E771 [41] and E789 [42]; cms-energy range: $[29.1: 38.8] \mathrm{GeV}$ ) and five cross section ratios $R_{\psi}=\sigma_{\psi(2 S)} / \sigma_{J / \psi}$ (cms-energy range: [20.6 : 29.1] GeV), for a total of 29 experimental results. This set of data shows a good overall consistency, except for the few measurements of $\sigma_{J / \psi}$ already mentioned in the previous section.

As a first step, the fit has been performed on all the 29 experimental results using the MRST2002nlo and the CTEQ6m PDFs. Since the charmonium production from singlet states strongly depends on the factorization $\left(\mu_{F}\right)$ and renormalization $\left(\mu_{R}\right)$ scales, we opted for determining the proper scale as an additional free parameter of the fit. The resulting optimal scale values are $\mu=1.5 \mu_{0}$ for MRST2002nlo 
and $\mu=2.6 \mu_{0}$ for CTEQ6m, where $\mu=\mu_{F}=\mu_{R}$ and $\mu_{0}=2 m_{c}$ with $m_{c}=1.5 \mathrm{GeV}$. The fit results obtained with these scale values are reported in Tab. 4.

\begin{tabular}{|l|c|c|}
\hline & MRST2002nlo & CTEQ6m \\
\hline$\mu_{F}=\mu_{R}$ & $1.5 \mu_{0}$ & $2.6 \mu_{0}$ \\
$\chi^{2} /$ d.o.f. & $114 / 27$ & $170 / 27$ \\
$\lambda_{J / \psi}$ & $0.089 \pm 0.013$ & $0.211 \pm 0.027$ \\
$\lambda_{\psi(2 S)}$ & $0.061 \pm 0.012$ & $0.112 \pm 0.017$ \\
\hline
\end{tabular}

Table 4: Results of the fit performed using the optimal scale factors for the MRST2002nlo and the CTEQ6m PDFs.

The $\chi^{2}$ of the fit is poor for both fits, since, as previously discussed, the measurements of $\sigma_{J / \psi}$ are not internally compatible. A reasonable explanation is that in some cases the experimental uncertainty might have been underestimated. With this assumption, we increased the uncertainties of the fit results by a scaling factor $(s=2.05$ and $s=2.51$ respectively), obtained following the PDG prescriptions [21]. The ratios of the matrix elements $\left(\lambda_{J / \psi}, \lambda_{\psi(2 S)}\right)$ provided by the fits differ by about a factor two mainly because of the different scales used. Nevertheless, in both fits the color octet matrix elements are found to be much smaller $(1 / 5-1 / 10)$ than those extracted at the Tevatron [17. Given that the CTEQ6m PDF shows a worse adaptation to the data, we have decided to choose the MRST2002nlo PDF fit as our baseline fit, while the results from the CTEQ6m PDF have been considered in the systematic uncertainties determination.

The fit results as a function of the cms-energy are displayed in Fig. 2 for the MRST2002nlo PDF. In the top plot the $J / \psi$ cross section is shown. In the bottom-left plot the $\psi(2 S)$ cross section is shown, while the $\sigma_{\psi(2 S)} / \sigma_{J / \psi}$ ratio is displayed in the bottom-right plot. The open circles in the two bottom plots represent the results calculated from the published papers and not used in the fit (see Tab. 3). The dot-dashed line indicates the NRQCD predictions without any octet contribution in the charmonium production, while the solid line is the result of the fit. The two dashed lines are obtained by independently varying the scales $\mu_{F}$ and $\mu_{R}$ between $\mu_{0}$ and $4 \mu_{0}$.

As one can see the curves obtained from the fit can well reproduce the general trend of the $J / \psi$ and $\psi(2 S)$ cross sections as a function of the cms-energy. In order to fit the data the production from singlet states seems to be not sufficient and contributions from octet states must be considered. However, the fit shows that the amount of octet production is small compared to the results from Tevatron data [17. and is in agreement the results on charmonium production obtained at HERA [5], where the octet contributions were negligible.

The stability of the results with respect to our baseline fit and the systematic uncertainties have been checked with detailed studies, mainly by changing the selection of the measurements to be fitted and the PDF. First we excluded the experimental results which show a bad partial $\chi^{2}\left(\chi_{p}^{2}>9\right)$ and consequently are not compatible with the NRQCD calculations. Following these prescriptions the four $J / \psi$ cross section measurements from the E331 [28], E705 [31], E595 34] and E771 [41] experiments were left out, obtaining a much improved $\chi^{2}\left(\chi^{2} / d o f=37 / 23\right)$, with only a few percent variation on $\lambda_{J / \psi}$ and $\lambda_{\psi(2 S)}$. Similar results have been obtained by excluding the four clearly incompatible measurements of $\sigma_{J / \psi}$ at 20.6, 23.8 and 24.3 GeV (E331 [28], E444 [29], E705 [31] and UA6 [32]) discussed in the previous section. More fits have been performed by changing the cms-energy range of the measurements and by using different target selections with the MRST2002nlo and the CTEQ6m PDF. Again the results showed a 

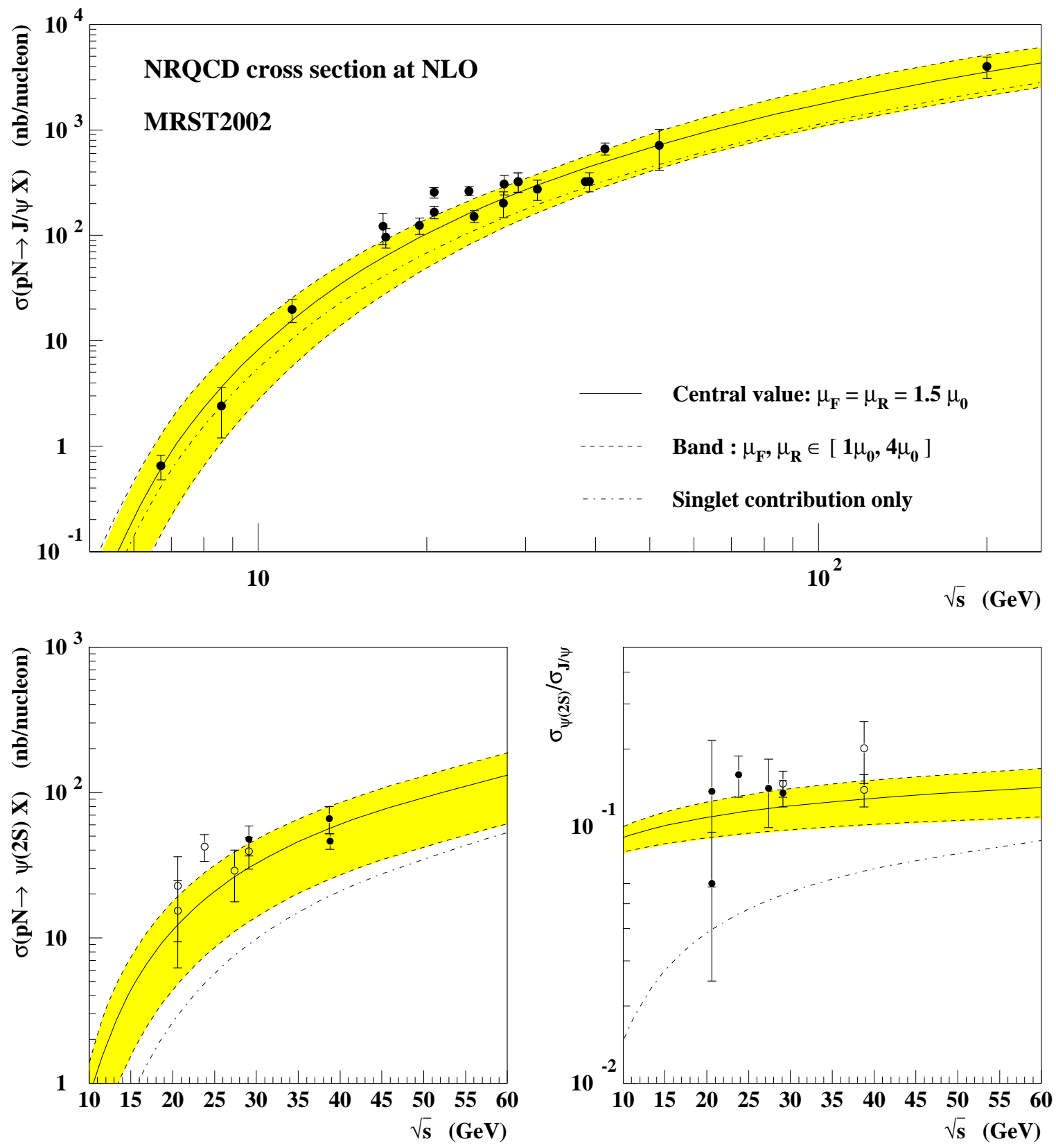

Figure 2: Fit results as a function of the cms-energy for the $J / \psi$ cross section (top), the $\psi(2 S)$ cross section (bottom-left) and the $\sigma_{\psi(2 S)} / \sigma_{J / \psi}$ ratio (bottom-right). The open circles in the two bottom plots represent the results calculated from the published papers which are not used in the fit.

very good stability on the cross sections; the only significant change observed is, as shown in Tab. 4 the dependence of the $\lambda_{J / \psi}$ and $\lambda_{\psi(2 S)}$ parameters on the actual scales $\left(\mu_{F}\right.$ and $\left.\mu_{R}\right)$ and PDF used. No significant variation of the results is observed when we include in the fit, in addition to $\sigma_{J / \psi}$ data, only $\sigma_{\psi(2 S)}$ or only $\sigma_{\psi(2 S)} / \sigma_{J / \psi}$ measurements. In the quoted uncertainties, the correlations between different 


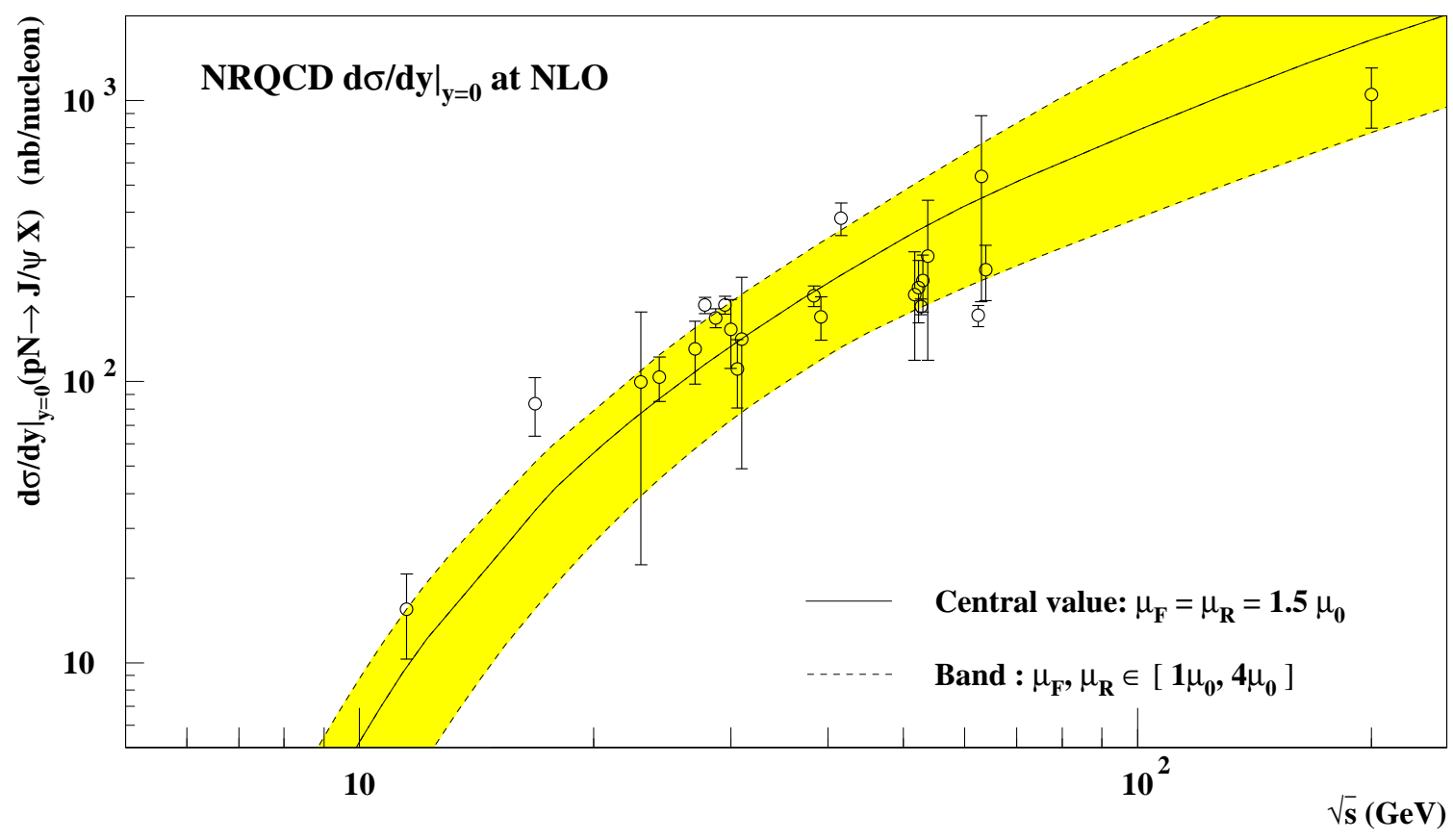

Figure 3: The $J / \psi$ differential cross section $d \sigma_{p N} / d y$ at $y=0$, as a function of the cms-energy. The data shown here are not used in the fit. The theoretical prediction with its uncertainty corresponds to the fit to the total cross sections, as shown in Fig. 2 ,

experimental results, due to the dilepton decay branching ratios and to the luminosity and efficiencies determinations for different measures performed by the same experiment, have been found to have a small impact.

Once the stability of the fit procedure and its precision have been established, one can obtain predictions for other quantities. As a first application, in Fig. 3. we show the comparison between the differential cross sections $d \sigma_{p N} / d y$ at $y=0$, as a function of the cms-energy, and the theoretical predictions at NLO. The data shown in the plot have not been used in the fit and therefore this result is an important check of the overall consistency of theory and experimental data. As a further application of our analysis, we present the predictions for the $J / \psi$ and $\psi(2 S)$ cross sections and their ratio at $\sqrt{s}=41.6 \mathrm{GeV}$, where HERA- $B$ [43] has obtained the most recent result on the $J / \psi$ cross section from a Minimum Bias data sample. The values obtained from the fit are the following:

$$
\begin{gathered}
\sigma_{J / \psi}=(502 \pm 44) \mathrm{nb} / \text { nucleon }, \\
\sigma_{\psi(2 S)}=(65 \pm 11) \mathrm{nb} / \text { nucleon }, \\
R_{\psi}=(0.130 \pm 0.019)
\end{gathered}
$$

where the quoted errors include the uncertainties due to the fit, to the data selection and to the PDF. The systematic uncertainties due to the PDF have been obtained following the so called "Les Houches Accord" prescriptions as discussed in Ref. [4]. 


\section{Conclusions}

In this paper we have collected all available data on $J / \psi$ and $\psi(2 S)$ production in hadron collisions (with the exception of data obtained in pion-nucleus collisions) and updated them in the light of the most precise determinations of nuclear effects. We have then presented their analysis in the context of NRQCD, using NLO predictions for the short-distance cross sections and fitting the color-octet nonperturbative matrix elements. In order to ease the comparison with the available determinations, we have chosen the values extracted at the Tevatron as our reference. We find sizeable systematic uncertainties associated both to the experimental data, which sometimes are marginally consistent among themselves, and to the fixed-order nature of theoretical predictions. Nevertheless, our results clearly indicate that the amount of color-octet contributions needed to explain fixed-target data is only about $10 \%$ of that fitted at the Tevatron. One can certainly argue that part of this discrepancy might be associated to the fact that the Tevatron analysis is based on LO calculations only. On the other hand, the difference is too large to be resolved by the inclusion of higher-order corrections. In addition, it is plausible to speculate that once the NLO corrections to the color-singlet production were computed and included in the analysis, there would be very little room left for color-octet contributions to fit the fixed-target experiments, in close analogy to what happens in photoproduction [5] and in agreement with the results in Ref. 49] where a different approach is used.

\section{Acknowledgments}

We would like to thank Mike Medinnis and many colleagues of the HERA- $B$ Collaboration for the support during this analysis and for many stimulating discussions.

\section{References}

[1] For a review, see N. Brambilla et al., "Heavy quarkonium physics", hep-ph/0412158.

[2] E. L. Berger and D. L. Jones, Phys. Rev. D 231521 (1981).

[3] R. Baier and R. Ruckl, Phys. Lett. B 102, 364 (1981).

[4] G. T. Bodwin, E. Braaten, and G. P. Lepage, Phys. Rev. D 51, 1125 (1995) [Erratum-ibid. D 55, $5853(1997)]$.

[5] M. Krämer, Prog. Part. Nucl. Phys. 47, 141 (2001).

[6] CDF collaboration, Note 05-04-28.

[7] M. Beneke and I. Z. Rothstein, Phys. Rev. D 54, 2005 (1996) [Erratum-ibid. D 54, 7082 (1996)] arXiv:hep-ph/9603400.

[8] F. Maltoni, hep-ph/0007003.

[9] G. P. Lepage, L. Magnea, C. Nakhleh, U. Magnea and K. Hornbostel, Phys. Rev. D 46, 4052 (1992).

[10] G. C. Nayak, J. W. Qiu and G. Sterman, hep-ph/0509021.

[11] F. Maltoni, M. L. Mangano and A. Petrelli, Nucl. Phys. B 519, 361 (1998). 
[12] A. Petrelli, M. Cacciari, M. Greco, F. Maltoni, and M. L. Mangano, Nucl. Phys. B514, 245 (1998).

[13] M. Krämer, Nucl. Phys. B 459, 3 (1996).

[14] W. Buchmüller and S. H. Tye, Phys. Rev. D24, 132 (1981).

[15] E. J. Eichten and C. Quigg, Phys. Rev. D52, 1726 (1995).

[16] F. Abe et al., (CDF Collaboration), Phys. Rev. Lett. 79, 572 (1997).

[17] P. Nason et al., hep-ph/0003142.

[18] H.L. Lai et al., Eur. Phys. J.12, 375 (2000).

[19] A.D. Martin et al., Eur. Phys. J.28, 455 (2003).

[20] G. T. Bodwin, E. Braaten and J. Lee, Phys. Rev. D 72 (2005) 014004.

[21] S. Eidelmann et al., Review of Particle Physics, Phys. Lett. B 592, 1 (2004).

[22] M.J. Leitch et al., (E866 collaboration), Phys. Rev. Lett. 84, 3256 (2000).

[23] A. Bamberger et al., Nucl. Phys. B 134, 1 (1978).

[24] M.J. Corden et al. (WA39 collaboration), Phys. Lett. 98B, 220 (1981).

[25] Yu.M. Antipov et al., Phys. Lett. 60B, 309 (1976).

[26] K.J. Anderson et al., Phys. Rev. Lett. 36, 237 (1976), Phys. Rev. Lett. 37, 799 (1976).

[27] J. Badier et al., (NA3 collaboration), Z. Phys. C 20, 101 (1983).

[28] J.G. Branson et al., (E331 collaboration), Phys. Rev. Lett. 38, 1331 (1977).

[29] K.J. Anderson et al., (E444 collaboration), Phys. Rev. Lett. 42, 944 (1979).

[30] J.H. Cobb et al., Phys. Lett. 68B, 101 (1977).

[31] L. Antoniazzi et al., (E705 collaboration), Phys. Rev. D 46, 4828 (1992).

[32] C. Morel et al., (UA6 collaboration), Phys. Lett. B 252, 505 (1990).

[33] H.D. Snyder et al., (E288 collaboration), Phys. Rev. Lett. 36, 1415 (1976).

[34] E.J. Siskind et al., (E595 collaboration), Phys. Rev. D 21, 628 (1980).

[35] M.C. Abreu et al., (NA51 collaboration), Phys. Lett. B 438, 35 (1998).

[36] M.C. Abreu et al., (NA38 collaboration), Phys. Lett. B 444, 516 (1998).

[37] B. Alessandro et al., (NA50 collaboration), Eur. Phys. J. C 33, 31 (2004).

[38] C. Kourkoumelis et al., Phys. Lett. 91B, 481 (1980).

[39] A.G. Clark et al., Nucl. Phys. B 142, 29 (1978). 
[40] A. Gribushin et al., (E672/706 collaboration), Phys. Rev. D 62, 012001 (2000).

[41] T. Alexopoulos et al., (E771 collaboration), Phys. Rev. D 55, 3927 (1997).

[42] M.H. Schub et al., (E789 collaboration), Phys. Rev. D 52, 1307 (1995).

[43] I. Abt et al., (HERA-B collaboration), submitted to Phys. Lett. B., hep-ex/0512029.

[44] E. Nagy et al., Phys. Lett. 60B, 96 (1975).

[45] E. Amaldi et al., Lett. Nuo. Cim. 19, 152 (1977).

[46] S.S. Adler et al., (PHENIX collaboration), Phys. Rev. Lett. 92, 051802 (2004).

[47] T.H. Chang et al.,(E866 collaboration), Phys. Rev. Lett. 91, 211801 (2001); T.H. Chang, PhD Thesis, hep-ex/0012034.

[48] J. Pumplin et al., JHEP 0207, 012 (2002).

[49] V. A. Khoze et al., Eur. Phys. J. C39, 163, (2005). 